\title{
À propos de Bambous kanak. Une passion de
} Marguerite Lobsiger-Dellenbach

Isabelle Leblic

\section{OpenEdition}

1 Journals

\section{Édition électronique}

URL : http://journals.openedition.org/jso/1882

DOI : 10.4000/jso.1882

ISSN : 1760-7256

\section{Éditeur}

Société des océanistes

\section{Édition imprimée}

Date de publication : 15 décembre 2008

Pagination : $311-317$

ISBN : 978-2-85430-012-3

ISSN : 0300-953x

\section{Référence électronique}

Isabelle Leblic, «À propos de Bambous kanak. Une passion de Marguerite Lobsiger-Dellenbach », Journal de la Société des Océanistes [En ligne], 126-127 | Année 2008, mis en ligne le 01 décembre 2008, consulté le 19 juin 2020. URL : http://journals.openedition.org/jso/1882 ; DOI : https://doi.org/ $10.4000 / j s 0.1882$ 


\section{MISCELLANÉES}

\section{À propos de Bambous kanak. Une passion de Marguerite Lobsiger-Dellenbach ${ }^{\mathbf{1}}$}

par

Isabelle LEBLIC*

Cet ouvrage collectif, publié à l'occasion de l'exposition du même nom au musée d'ethnographie de Genève (MEG), fait office de catalogue de l'exposition. Dirigé par la commissaire de l'exposition, Roberta Colombo Dougoud, il rassemble les contributions de huit spécialistes du sujet: outre cette dernière (conservatrice du département Océanie du MEG), Roger Boulay (chargé de mission pour les collections océaniennes à la direction des Musées de France - DMF), Diane Cousteau (doctorante en anthropologie de l'art, notamment kanak), Henri Gama (conseiller en Arts visuels), Carole Ohlen (attachée de conservation au musée de NouvelleCalédonie), Marie-Claude Tjibaou (présidente du conseil d'administration de l'Agence de développement de la culture kanak - ADCK) et Lorin Wüscher (ethnologue et historien de l'art ayant participé à la conception de l'exposition).

Construit en trois parties (1. Marguerite Lobsiger-Dellenbach et les bambous gravés, pp. 17-46; 2. Les bambous kanak, pp. 47-96; 3. La collection de vingt-neuf bambous kanak du MEG, pp. 97-181), ce «Beau-Livre» s'ouvre sur un avant-propos de Marie-Claude Tjibaou (pp. 7-9), une préface du directeur du MEG, Jacques Hainard (pp. 10-11) et une introduction de Roberta Colombo Dougoud et Lorin Wüscher (pp. 12-13) qui ont conçu l'exposition.
Marie-Claude Tjibaou commence par quelques souvenirs personnels à propos des bambous et de leurs diverses utilisations... Quand adolescente, avec ses cousines, elle parcourait les bambouseraies - à Ponérihouen, on dit «bambounières »- bordant la rivière de Pwârâiriwâ, où se trouvaient de nombreux messages codés gravés sur les troncs par de jeunes amoureux ou bien tout simplement la trace d'une traversée de la bambouseraie. Quiconque connaît la Nouvelle-Calédonie sait ce qu'il en est de ces multiples " graffitis » sur les troncs d'arbres de toutes sortes que l'on trouve au gré des pérégrinations dans les forêts, le long des routes ou dans les cocoteraies... Marie-Claude nous parle aussi des radeaux en bambous utilisés pour traverser la rivière ou bien pour une partie de pêche, « aux moules avec [ses] aînées ». Aujourd'hui encore, la rivière est bordée de nombreuses bambouseraies et il n'est pas rare de voir ces radeaux accostés le long de ses rives. Enfin, elle nous parle du bambou utilisé pour transporter l'eau: « canalisation ouverte [...] supportée par des fourches piquées au sol » pour amener l'eau de la montagne aux habitations, qu'il fallait nettoyer et entretenir régulièrement, notamment après chaque grande pluie, au risque de ne plus avoir d'eau potable... On trouve également de telles canalisations dans les tarodières irriguées,

1. Ouvrage dirigé par Roberta Colombo Dougoud et paru en 2008 à Genève (MEG, INFOLIO, coll. Sources et témoignages 9 , 184 p., bibliogr., glossaire, nombreuses illustrations (photographies en couleur ou noir et blanc, dessins au trait), pour accompagner l'exposition au musée d'Ethnographie de Genève.

* Ethnologue, LACITO-CNRs, Villejuif, leblic@vjf.cnrs.fr 

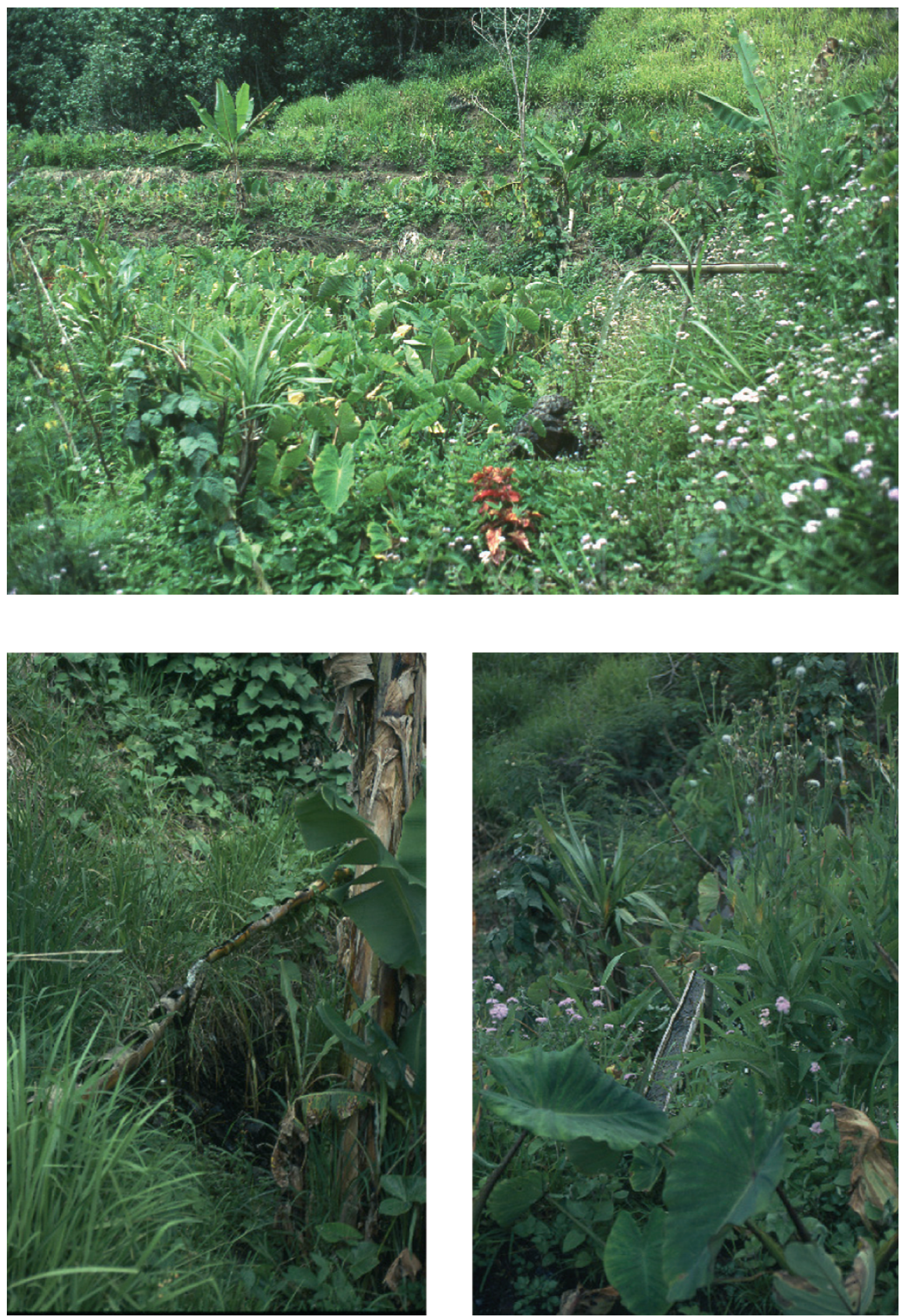

Pнотоs 1 à 3. - Exemple de l'utilisation de bambous dans des tarodières de Canala en 1985 (clichés d'Isabelle Leblic) 
comme j'ai pu le voir moi-même en service à Canala dans les années 1980 (voir photos). Mais le bambou est aussi objet d'ornement, peigne à cheveux, plus ou moins gravé, ou encore instrument de musique, au plus simple souvent, juste un tronc pilonnant le sol pour marquer le rythme des danses des célèbres pilous dans les célébrations de toutes sortes (Nouvel An, mariages ou levées de deuil) :

« Nous étions alors emportés, transportés dans un tourbillon, soulevant des nuages de poussière et nous tournions, tournions, comme aspirés dans une spirale qui nous emmenait jusqu'aux premières lueurs du jour. » (p. 7)

C'est tout cela la réalité passée (et encore souvent actuelle) du bambou kanak. C'est aussi aujourd'hui (et sans doute hier) des poteaux dans diverses constructions, des parois de case, des tuteurs dans les plantations, des cannes à pêche et jusqu'à toutes sortes d'objets utilitaires actuels (des dessous-de-plat, des cadres, etc.), et j'en oublie sûrement...

Et, à côté de cela, se trouvent les fameux bambous gravés. D'hier à aujourd'hui avec ceux de l'artiste kanak «Micheline Néporon, la tresseuse d'histoire ", qui grave à nouveau des bambous (depuis 1992) et dont Henri Gama nous parle dans cet ouvrage (pp. 91-96). C'est, nous rappelle Marie-Claude Tjibaou, par le Festival Mélanésia 2000 dont son mari Jean-Marie fut l'un des principaux concepteurs, que le bambou gravé a refait son entrée dans l'art contemporain kanak. Elle nous dit à ce propos :

«"Mélanésia 2000", premier festival des arts mélanésiens de Nouvelle-Calédonie qui s'est tenu en 1975 à Nouméa, fut sans conteste l'acte fondateur de la renaissance de la culture kanak. Né d'une revendication identitaire, ce mouvement a suscité au sein de la société kanak une prise de conscience de la richesse bien vivante de sa propre culture. » (p. 8)

Aujourd'hui, les polyptyques de Micheline Néporon ${ }^{2}$, aux motifs « peints à l'encre de Chine dans le style des bambous gravés, mais avec des pinceaux de calligraphe », racontent des histoires de la société kanak actuelle, les étapes de la vie de la naissance à la mort, les transformations subies par cette société, comme hier les bambous gravés en racontaient d'autres, des scènes de pêche, des pilous ou des cérémonies de toutes sortes... et qui ont traversé le temps, comme sup- port de mémoire et de récits illustrant les divers aspects de la vie quotidienne des Kanak jusqu'à l'irruption de la colonisation et de ses premières conséquences (il semblerait que leur production ait été arrêtée vers 1917, date d'une des dernières et célèbres révoltes des Kanak contre le système colonial $^{3}$ ).

Selon Maurice Leenhardt (1937: 109-111), ces bambous gravés étaient utilisés comme bâtons de voyage tout en gardant trace des hauts faits ou malheurs de leurs ancêtres (voir Lambert, $1980: 68$ ), « viatique pour se protéger des dangers de la route lorsqu'ils s'aventuraient hors de leur village » (p. 12) car, contenant, comme le rappelle Marie-Claude Tjibaou, les herbes protectrices propres à chaque clan, souvent nommé « médicaments » en français local, dont le pouvoir est lié aux esprits ancestraux dits totems. Il est vrai que les Kanak des temps passés parcouraient de longues distances pour se rendre d'un lieu à un autre, pour entretenir leurs réseaux d'alliance, fuir un ennemi ou faire la guerre et qu'ils ne se séparaient jamais de leurs « feuilles » protectrices.

Ces bambous sont tout à la fois, comme nous l'évoque Jacques Hainard dans sa préface, des « objets de mémoire et de transmission » et « un trait d'union » entre le passé et le présent et entre les cultures, celle des Kanak, mais aussi celle de la colonisation, puisqu'ils présentent aussi « des scènes et des usages relatifs à l'histoire de la colonisation de la Nouvelle-Calédonie » (p. 10). Ils sont en cela, en quelque sorte, une trace écrite - dans ces sociétés à tradition orale -, une bande dessinée de la perception par les Kanak de l'irruption des Européens dans leur pays, mais aussi une illustration de leur vie quotidienne (modes de subsistance, architecture, mythes et rituels). $\mathrm{Ou}$, comme on a dit ailleurs ( $c f$. Nathan Wachtel en 1971), la vision des vaincus ${ }^{4}$ :

« Marguerite et Georges virent dans les bambous gravés un mode d'expression qui intégrait une vision autochtone de la colonisation. Pour eux, ces œuvres permettaient de saisir le point de vue des Kanak, leur interprétation de leur culture et de l'arrivée du colonisateur. » (p. 28)

« La mémoire est l'avenir du passé », selon Paul Valéry cité en exergue de l'introduction de cet ouvrage (p. 13) et comme le disait si bien Jean-Marie Tjibaou, en conclusion de son entretien dans Les temps modernes:

2. Le MEG a enrichi sa collection ancienne par l'acquisition de quatre bambous gravés exécutés par cette artiste kanak contemporaine.

3. Nous verrons par ailleurs ci-dessous le bambou inédit de Gustave Glaumont trouvé au musée de Nantes dont nous parle Roger Boulay dans cet ouvrage (pp. 85-90), qui semble garder une trace de la précédente révolte d'importance, celle de 1878.

4. « [...] l'anthropologue René Verbeau [...], dans L’homme, races et coutumes (1931 : 335), avait comparé les bambous kanak aux codex peints du Mexique destinés à rappeler de grands événements de l'histoire mésoaméricaine » (p. 28). 
«Le retour à la tradition, c'est un mythe; je m'efforce de le dire et de le répéter. C'est un mythe. Aucun peuple ne l'a jamais vécu. La recherche d'identité, le modèle, pour moi, il est devant soi, jamais en arrière. C'est une reformulation permanente. Et je dirais que notre lutte actuelle, c'est de pouvoir mettre le plus possible d'éléments appartenant à notre passé, à notre culture dans la construction du modèle d'homme et de société que nous voulons pour l'édification de la cité. [...] Notre identité, elle est devant nous $^{5}$. » $(1985: 1601)$

Ces bambous gravés sont ainsi la mémoire d'un passé révolu transmis aux générations futures, dont la prise en compte est importante aujourd'hui pour la construction de l'avenir du pays au moment où l'on cherche à définir une nouvelle citoyenneté.

Dans leur introduction, Roberta Colombo Dougoud et Lorin Wüscher nous rappellent le trajet de Marguerite Lobsiger-Dellenbach, directrice du MEG pendant quinze ans (19521967), qui se consacra à plusieurs passions dont celle pour les bambous gravés kanak (kârè a tâ, en langue ajië). Ils nous précisent également que les bambous présents dans les musées furent « récoltés entre 1850 et 1920 » alors que « leur production date principalement du $\mathrm{XIX}^{\mathrm{e}}$ siècle » (p. 12). Avec ses vingt-cinq bambous, le MEG possède la seconde collection en importance, la première étant celle du musée du quai Branly de Paris, pour environ deux cents spécimens dispersés dans le monde entier. Certains d'ailleurs « refont surface» encore aujourd'hui, comme nous le raconte Christian Coiffier ci-dessous (voir p. x). Ils insistent sur la mémoire commune qui lie désormais les Kanak et le MEG grâce à la passion pour les bambous gravés de Marguerite Lobsiger-Dellenbach et ils présentent, à l'heure des revendications autochtones si nombreuses, l'exposition et l'ouvrage qui l'accompagne comme une forme de restitution symbolique.

Avant de rentrer plus avant dans ce livre, il nous faut en souligner l'agréable présentation, la mise en page de qualité sur un beau papier glacé. La reprise de dessins au trait en couleur sur des fonds violacés sur une page en début d'article après une reproduction partielle d'un bambou en double-page comme intercalaire entre deux parties du livre sont du plus bel effet, de même que les pages intercalaires entre deux articles détaillant telle partie d'un bambou. On peut regretter toutefois l'absence de reproduction « en déroulé » des bambous...

La première partie de ce livre nous présente Marguerite Lobsiger-Dellenbach et sa passion pour les bambous gravés kanak, à travers deux textes, l'un des organisateurs de l'exposition, Roberta Colombo Dougoud et Lorin Wüscher (Marguerite Lobsiger-Dellenbach et les bambous kanak : une femme, une passion un peuple, pp. 17-36), l'autre de Marguerite elle-même (Les gravures sur bambous de Nouvelle-Calédonie : un testament illustré d'une culture disparue, pp. 37-46). Le premier texte est très éclairant sur la femme " d'origine modeste » que fut Marguerite, née le 9 juillet 1905 à Genève « d'une mère genevoise et d'un père alsacien » (p. 17). Le décès de celui-ci alors qu'elle n'avait que onze ans l'empêcha de poursuivre ses études et elle dut aider sa mère au plus vite en travaillant comme chapelière ou sténodactylo dans un bureau d'avocat, puis au laboratoire d'anthropologie de la rue Saint-Victor auprès du professeur Pittard en juin $1922 \ldots$ C'est de là que naquit sa passion pour l'anthropologie, grandement encouragée par " le patron» comme elle l'appelait. Je ne reviendrai pas ici sur l'ensemble de son parcours, laissant le soin au lecteur de le découvrir au fil des pages. Disons juste comment elle en vint à se passionner pendant plus de trente ans pour les bambous gravés kanak, alors que, femme de nombreux terrains, elle ne se rendit jamais en Nouvelle-Calédonie. "C'est Eugène Pittard qui l'avait encouragée à les étudier », qui lui aurait dit : " vous devriez regarder de près ces bambous » (p. 27) et c'est ce qu'elle fit pendant une trentaine d'années. Son apport fut, dès ses premiers écrits, de se distancier de la vision purement décorative souvent mise en avant (voir entre autres Luquet, $1926: 41$ ) et de considérer les bambous gravés comme des « documents ethnographiques de grande valeur et certaines fois des témoignages psychologiques de premier ordre » (p. 32). Malgré tout, ses écrits sont marqués par les idées de leur époque et Marguerite Lobsiger-Dellanbach, malgré son profond respect pour les Kanak, sont «empreints d'une certaine condescendance à l'égard de ce peuple opprimé » (p. 32).

Ainsi, «le Kanak lui apparaissait surtout comme un être prisonnier de ses coutumes, dont "toute la vie psychique [...] est limitée par la crainte des morts et de leur esprit, par le souci de ne pas violer les tabous. [...]" ". Ce qui fait que le graveur kanak ne disposant pas de libre-arbitre, ne peut que « retranscrire des schémas qui lui sont dictés par la tradition » (p. 32).

Puis, la reproduction de l'article de Marguerite et Georges Lobsiger-Dellenbach, paru initialement dans le Courrier des messageries mari-

5. Cette phrase est reprise dans de nombreuses publications. On peut regretter qu'elle soit présentée ici tronquée par les auteurs (p. 13) sans qu'ils aient noté les coupes faites par des points de suspension... 
times 105 (juillet-août 1968) - revue sans doute peu connue du milieu de la recherche -, permet au lecteur non averti comme au spécialiste de la Nouvelle-Calédonie de découvrir leur travail de recherche sur les bambous gravés. Pourvu de douze illustrations (dessins au trait) et d'une reproduction d'une partie de bambou en double page, il nous présente les bambous kanak comme une partie de "l'héritage des peuples sans écriture qui inventèrent des procédés originaux pour conserver le souvenir de leur vie quotidienne et pour inscrire à jamais les espoirs et les craintes de ce monde tout autant que l'appel à la protection des dieux et des esprits », et conclut sur le fait que :

« Le silence des musées n'est pas celui des tombes, mais celui qu'impose l'amical respect pour le testament illustré d'une culture disparue. » (p. 43)

Heureusement, il n'en est rien, la culture kanak est bien vivante !

La deuxième partie de cet ouvrage comporte cinq textes. Carole Ohlen étudie l'art traditionnel du bambou gravé kanak (pp. 47-68). Illustré de dix-huit dessins au trait et de deux photos de partie de bambou, cet article revient sur leurs caractéristiques : les divers emplois du bambououtils, instrument de musique, contenant, etc. - ; leurs traces dans les écrits et la tradition orale ; la fonction des gravures ; leur fabrication et technique de gravure ; la tradition ancienne des décors face aux scènes de la vie coloniale...

Diane Cousteau évoque ensuite les bambous gravés dans la Nouvelle-Calédonie contemporaine (pp. 69-81). Elle nous montre comment ce mode d'expression est réactualisé aujourd'hui par de nombreuses pratiques artistiques (les jeunes artistes kanak tels, par exemple, Paula Boi, Yvette Bouquet et Micheline Néporon...) ou simplement décoratives (grilles et murs du musée de Nouvelle-Calédonie, péage de la route territoriale 1 à la sortie Nord de Nouméa, fuselage des avions d'Air Calédonie...). Après avoir précisé que ces objets « étaient des possessions personnelles, dont la création ne semble avoir été liée à aucun statut social particulier ${ }^{6}$ et dont la transmission se faisait en dehors des échanges coutumiers, dans le contexte d'un don ou d'un héritage entre deux individus ", elle insiste sur le fait qu'ils sont des objets " emmêlés » selon la définition de Thomas (1991), matérialisant la rencontre de deux cultures et à la charnière entre art traditionnel et art contemporain (p. 69), Elle pose même la question de leur réelle existence avant l'ère coloniale que « très peu d'éléments attestent » :

« bien que la tradition orale fasse remonter cet art à une période antérieure à l'arrivée des Européens, aucun exemple concret ne vient appuyer cette hypothèse avec certitude. » (p. 70)

En rapprochant les bambous gravés, comme je l'ai fait plus haut, des graffitis végétaux des bords de routes, Diane Cousteau reprend là une idée avec laquelle on ne peut que souscrire quand on connaît bien les paysage de la NouvelleCalédonie kanak. Comme le souligne YvesBéalo Gony dans ces pages, mais aussi MarieClaude Tjibaou dans sa préface, c'est là une pratique ancienne :

« les jeunes kanak ont de tout temps incisé ces plantes pour communiquer entre eux, notamment pour déclarer leur flamme et se donner rendez-vous, à l'insu des adultes. » (p. 71)

Dans les créations actuelles, les artistes vont de la représentation de thèmes traditionnels de la vie en tribu (Clarisse Itréma) pour assurer une transmission des traditions, à un subtil mélange des deux avec l'irruption d'avions ou d'HLM dans les scènes d'échanges coutumiers (Chanel Cinedrawa $)^{7}$, pour en arriver à une rupture totale lorsque l'artiste utilise le support du bambou gravé pour un message contemporain d'émancipation (Stéphanie Wamytan), avec des représentations à caractère explicitement sexuel bien éloignées des codes traditionnels et jugés par plus d'un scandaleuses. Diane Cousterau nous rappelle que personne mieux que les descendants des créateurs, "qui connaissent l'histoire de leur clan et peuvent nous indiquer les événements auxquels les illustrations font référence » (p. 75) n'est plus à même de nous éclairer sur leurs significations. Et cela grâce au rapatriement virtuel via des photographies de l'un de ces bambous, le vingt-cinquième du catalogue, qu'elle montre à l'île des Pins, en suivant la démarche initiée entre autres par James Clifford pour des objets tlingit de la collection Rasmussen, par Amiria Henare pour des tissages maoris ou encore par Nicholas Thomas et John Pule pour les Hiapo de Niue. L'analyse qui est produite, suite à la discussion avec les Kwênyii à propos de ce bambou, est des plus intéressantes et permet de confirmer ce dont on pouvait se

6. Nous savons en effet que de nombreuses techniques sont appropriées par des clans particuliers (clans « pêcheurs » pour les engins de pêche et de navigation, clans « potiers ", etc.) et cette information est donc intéressante à noter.

7. Montrant, si ce n'est le métissage culturel de ces pratiques, du moins l'intégration d'éléments exogènes dans des pratiques dites traditionnelles, comme nous l'avons montré dans divers domaines à plusieurs reprises dans nos écrits sur les transformations techniques notamment (voir entre autres Leblic, 2008). 
douter quand on connaît l'île des Pins ${ }^{8}$, à savoir le caractère de document historique de ce bambou particulier mais aussi des gravures sur bambou en général :

« lues de haut en bas, elles constituent une description plus ou moins chronologique des événements qui se sont déroulés à l'île des Pins durant les trois derniers quarts du XIX ${ }^{\mathrm{e}}$ siècle, détaillant les nouveautés qui ont été introduites. » (p. 75)

Ainsi, ce bambou nous raconte la vie traditionnelle avec un couple de Kanak en tenue traditionnelle, le bagayou pour l'homme et la jupe de fibre pour sa compagne et une scène maritime avec une pirogue traditionnelle, des miikwa (Chanos chanos Forsskål), puis viennent les transformations dues à l'arrivée des Européens marquées par une maison de style européen, une église et le bateau HSM Camden qui amena à l'île des Pins les déportés de la Commune, etc. L'analyse de ce bambou est particulièrement fouillée et donne envie que ce travail puisse être généralisé à l'ensemble des bambous gravés dont on a connaissance pendant qu'il est temps de le faire, si tant est que la mémoire orale de l'histoire du pays kanak s'estomperait avec les années qui passent - fait dont personne ne peut être sûr, mais qui est souvent affirmé en NouvelleCalédonie. Comme le conclut l'auteur :

«Il importe peu de savoir si les interprétations kanak des bambous gravés sont correctes ou non. L'essentiel est que ces interprétations indigènes puissent naître, et que la réflexion sur ces œuvres puisse être stimulée au niveau local et non plus simplement projeté de l'extérieur. » (p. 79)

Reste à avoir suffisamment de renseignements sur les provenances des diverses pièces conservées dans les collections pour que de tels rapatriements virtuels puissent s'avérer fructueux.

Roger Boulay revient de son côté, d'une part, sur la source d'information que représente chaque bambou gravé (À y regarder de plus près..., pp. 81-84) et, d'autre part, sur un bambou inédit de la collection Gustave Glaumont au musée d'Histoire de Nantes (Et si les bambous gravés kanak racontaient l'histoire?..., pp. 85-90). Dans le premier texte, il attire notre attention sur un procédé peu décrit, le tirage du poteau central de la case, en insistant sur le fait que ce n'est pas qu'un geste technique, mais aussi un des points centraux de la construction de toute case. Et là encore, je ne peux m'empêcher de faire le rappro- chement avec le domaine maritime que je connais bien et le tirage des bois de coques des pirogues (Leblic, 2008), dont le transport est souvent aussi long et difficile que celui qu'expose ici Roger Boulay, mobilisant autant les solidarités et forces physiques des humains que les ancêtres et leur puissance (p. 81). La comparaison de dessins de divers bambous, à propos du tirage du poteau central avec celui de Boesou Erijisi reproduit par Maurice Leenhardt ou avec une scène de bambou sur la pêche à la tortue permet de montrer comment les transports essentiels respectent tous le même principe, avec « une hiérarchisation des fonctions autour de l'objet à bouger " qui semble plus importante finalement que l'« organisation technique, ce que les différences d'échelle dans les personnages représentés pourraient figurer » (p. 83).

Dans son deuxième texte, Roger Boulay présente la découverte de la collection Glaumont aux pièces inédites comprenant le bambou gravé dont il est ici question. Mais avant cela, il revient sur ce que Gustave Glaumont avait écrit à propos des bambous :

« [...] les Calédoniens ne possèdent point l'écriture dans le vrai sens du mot; le seul système graphique dont ils aient connaissance n'est qu'une composition d'images, de vrais dessins : c'est la reproduction plus ou moins grossière, d'un arbre, d'une maison, d'un poisson, d'un oiseau, etc., ou un assemblage de traits horizontaux, verticaux, en losange, en carré, en cercle en étoile. Ces images désignent grossièrement et vaguement un événement qui a frappé leur imagination de manière profonde : un pilou pilou, une guerre, un naufrage, l'arrivée des Blancs et des premiers chevaux [...]. Autant que l'on puisse l'assurer, toutes ces images grossières ont l'intention de raconter, de dépeindre l'arrivée des premiers Blancs, des missionnaires, des grands navires et l'introduction des fusils et des chevaux; cette écriture se déchiffre peut-être, mais ne s'articule pas ; généralement, on doit commencer la lecture de bas en haut. » (p. 87)

Ce qui fait dire à Boulay que « cette dernière observation est absolument unique » et « qu'elle pourrait servir de proposition à l'étude systématique de ces objets dont la lecture, motif par motif, prévaut depuis Luquet qui tenta une première interprétation de ces scènes » (p. 87). La description de ce bambou inédit, dont la scène et son agencement sont uniques parmi les deux cents et quelques bambous connus, d'après Boulay, laisse à penser qu'il s'agit là d'un événement singulier, lié à des opérations militaires mobili-

8. J'ai fait la lecture de cet ouvrage en commençant par la fin pour admirer en premier lieu les bambous de la collection du MEG et, à la vision de ce bambou, j'avais plusieurs idées de rapprochements que j'ai retrouvées par la suite dans cette analyse (la maison de la reine Hortense, l'église, la pêche au miik $\boldsymbol{w} \boldsymbol{a}$, poissons coutumiers de l'île des Pins, en pirogue notamment). Je me demandais même si je ne devais pas demander l'autorisation de reproduire ce bambou dans l'ouvrage qui vient de paraître à la Société des Océanistes sur la pêche à l'île des Pins (cf. Leblic, 2008). 
sant troupes françaises et auxiliaires kanak (p. 89). Comme Gustave Glaumont débarqua en Nouvelle-Calédonie juste deux ans après les événements de juin 1878 (révolte qui embrasa toute la région allant de Canala à La Foa) marqués par la mort du grand chef Ataï tué par des auxiliaires kanak, c'est à juste titre que Roger Boulay se demande si ce bambou ne raconterait pas l'histoire de la révolte de 1878.

Enfin, Henri Gama termine cette section en présentant «Micheline Néporon, la tresseuse d'histoire » (pp. 91-96), qui peint, dessine et grave des bambous. Le MEG a d'ailleurs enrichi sa collection kanak de bambous gravés par l'acquisition de quatre de ses créations.

La troisième partie, rédigée par Roberta Colombo Dougoud et Lorin Wüscher, présente la collection des vingt-neuf bambous gravés du MEG (Catalogue de la collection du MEG avec photographies et notices détaillées, pp. 97-181) et la bibliographie de Marguerite LobsigerDellenbach sur les bambous kanak (pp. 182183). Il faut souligner la richesse de l'iconographie de ces quatre-vingt-quatre pages qui permet à chacun d'admirer ces bambous et d'entrer dans les scènes de la vie kanak qui y sont gravées. Sont ainsi présentées soixante-seize photographies, reproductions de bambous en grande majorité, mais aussi d'autres objets tels qu'une flèche faîtière, un chambranle de porte de case, un modèle réduit de case, un battoir de danse, un doigtier ou propulseur, une hache ostensoir, une coiffe cérémonielle ou un casse-tête à bec d'oiseau... et quarante-cinq dessins de motifs à plat par Philibert de Chastonay, du MEG - dont certains non numérotés (seize apparaissant seulement sous le numéro d'inventaire de l'objet ${ }^{9}$ ) et d'autres référencés en ill. $(8,15,16,23,24,25,26,35,38,41$, $52,56,61,62,64,66,78,79,81$ ) ou en fig. (1 à 9).

Néanmoins, on doit regretter le certain flou dans la façon de numéroter les illustrations (reproduction en photographies couleur des bambous ou bien les dessins des motifs à plat par le dessinateur du MEG), parfois numérotés à la suite et parfois non ${ }^{10}$. La présentation n'est pas très claire pour le lecteur et rend l'ouvrage peu commode par manque de liste finale des illustra- tions ou d'index, ce qui est malheureusement toujours le cas des catalogues d'exposition. Dommage ! On aurait aimé pouvoir, grâce à de telles listes, retrouver sans être obligé de feuilleter tout l'ensemble, tel ou tel bambou représentant une scène de pêche ou autre...

À plusieurs reprises, des bambous marquant très clairement les débuts de la colonisation rappellent le jugement très sévère et le peu d'intérêt de Marguerite et Georges Lobsiger-Dellenbach pour des objets qui ne seraient pas, selon eux, authentiques car non exempts d'influence extérieure. Cela nous renvoie à d'autres débats bien actuels sur l'authenticité et la tradition. Nous pourrions dire simplement que doit être considéré comme authentique ou traditionnel ce qui provient de la société considérée et est pensé comme tel par ses membres. Il s'agit peut-être d'une vision endogène de la question, mais ne serait-ce pas une des plus profitables ici ?

En guise de conclusion, je dirais que nous avons là cent quatre-vingt-quatre pages d'un voyage passionnant dans l'univers des bambous kanak, richement illustré et documenté, qui consolera tous ceux et celles qui ne pourront se rendre au MEG pour voir l'exposition qui a été le prétexte de cet ouvrage.

\section{BIBLIOGRAPHIE}

Entretien avec Jean-Marie Tjibaou, Les temps modernes 464 : Nouvelle-Calédonie : pour l'indépendance, pp. 1587-1601.

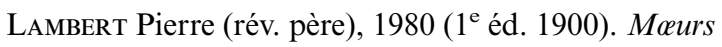
et superstitions des Néo-Calédoniens, Nouméa, Publications de la société d'études historiques de Nouvelle-Calédonie 14.

LEBLIC Isabelle, 2008. Vivre de la mer, vivre avec la terre... en pays kanak. Savoirs et techniques des pêcheurs kanak du sud de la Nouvelle-Calédonie, Paris, Société des Océanistes (avec le concours du musée de Nouvelle-Calédonie). Travaux et documents océanistes 1, $288 \mathrm{p}$.

LEENHARDT Maurice, 1937. Gens de la Grande Terre, Paris, Gallimard.

9. Pour chaque bambou de la collection, on trouve son numéro d'inventaire, sa provenance et son année d'acquisition, ses dimensions et sa description (couleur, nature des dessins et scènes représentées avec l'analyse qui en a été faite par Marguerite Dellenbach et Georges Lobsiger).

10. Ainsi, par exemple, pages 162-163, les dessins au trait ne sont indiqués que comme 22 MEG Inv. ETHOC 04I 748 (p. 162) et les photos comme ill. 76 et 77 (p. 163), alors qu'ailleurs on trouve des dessins au trait numérotés en illustrations comme page 153 (ill. 62 et 64). En fait, il semble que seuls les dessins au trait « déroulés » ou les reproductions de l'ensemble dessiné sur un bambou de l'inventaire du MEG ne soient pas numérotés et que tous les dessins de détails le soient. Mais cela n'est pas toujours visible à la lecture de l'ouvrage. 